\title{
Effect of Otago Exercise versus Dual Task Net Step Exercise on Balance and Functional Mobility in Community Dwelling Elderly Person with Knee Osteoarthritis - A Randomised Control Trial
}

\author{
Raymol Dagani Mohan Rao', Archana D. Methe ${ }^{2}$, Harshada Patil ${ }^{3}$, \\ Ronald Prabhakar ${ }^{4}$
}

\begin{abstract}
${ }^{1}$ B.P.Th. Intern, MMC College of Physiotherapy, Wanless Hospital, Miraj, Maharashtra, India
${ }^{2}$ Assistant Professor, MMC, College of Physiotherapy, Wanless Hospital, Miraj, Maharashtra, India

${ }^{3}$ Associate Professor, MMC, College of Physiotherapy, Wanless Hospital, Miraj, Maharashtra, India

${ }^{4}$ Professor, MMC, College of Physiotherapy, Wanless Hospital, Miraj, Maharashtra, India
\end{abstract}

\begin{abstract}
Background: Osteoarthritis (OA) is a chronic disease that can change the lifestyle of the affected person by producing pain and decreasing function; OA can result in loss of mobility, pain, disability, and dependence. Impaired balance and increased postural sway (oscillating movements of the body over feet during relaxed standing) both occur with advancing age. Diminished strength is well documented in the elderly.

Objective: The purpose of this study was to evaluate the effectiveness of Otago exercise versus dual task net step exercise on balance and functional mobility in community dwelling elderly person with knee OA.

Methods: Subjects with knee OA having balance and functional mobility problems were included in this study. Total of 36 both male and female were selected. Subjects those matching the inclusion criteria with age group 60-70 were included for the study. They were randomly divided into two groups; 18 subjects were present in each group. For 4 weeks and 3 days per week. Visual analogue scale, Berg Balance Scale, Time Up and Go test was used to assess the pre and post effects of exercises.

Result: Group A showed significant improvement in the balance and functional mobility as compared to group B .So the p-value $=0.000<.05$.

Conclusion: The study concludes that Otago exercises are more effective than the Dual Task Net Step Exercises in improving balance function and functional mobility in community dwelling elderly persons with knee respectively.
\end{abstract}

Keywords: Osteoarthritis, Otago Exercise, Dual Task Net Step Exercise, Berg Balance Scale, Time Up and Go test.

\section{INTRODUCTION}

Osteoarthritis (OA) is the major chronic musculoskeletal condition leading to disability worldwide. ${ }^{[1]}$ It is on the top of the list causing activity limitation and absent among working-age adults and is associated with a significant decline in function among older individuals. ${ }^{[1]}$ The knee being the most common weight bearing joint, $10 \%$ to $15 \%$ of all adults aged over 60 with the prevalence higher among women than men.

In India the prevalence is $28.7 \%$ with the medial compartmental OA being 
the commonest cause of disability. ${ }^{[2]}$ In rural Maharashtra the $21.6 \%$ of the population is affected with knee OA. Common signs and symptoms of knee OA include knee pain, joint stiffness, decreased muscle strength, and proprioceptive deficits. ${ }^{[4]}$ In addition; individuals with knee OA often exhibit poor neuromuscular control, slower walking speed, decreased functional ability, and an increased susceptibility to falling. ${ }^{[5]}$

Most common predictors of falls are abnormalities in gait or balance and a past history of fall. [6] Deficit in the motor components of balance is caused by musculoskeletal (i.e., poor posture, joint ROM limitations, and decreased muscle performance), When OA affects weightbearing joints, mainly the knee, it shows distinct reduction of muscle function which results to a reduction of balance and especially of the ability to perform sit-tostand tasks, to gait alterations, functional limitation, and loss of independence. ${ }^{[7]}$ That is why, OA is observed as an intrinsic risk factor for the event of falls. ${ }^{[8]}$

Osteoarthritis (OA) is a chronic disease that can result in loss of mobility, pain, disability, and dependence. ${ }^{[9]}$ Onethird of individual older than age 65 have radiographic evidence of OA. ${ }^{[10]}$

Knee pain is the prime symptom of OA among the elderly, which is seen in both men and women. To direct the progression of the knee OA assessment of pain intensity is used primarily as an outcome measure and Several scales were commonly used for the assessment of pain intensity. ${ }^{[11]}$ Among them, the VAS is frequently used in clinical practice. This pain-rating scale had shown good validity and reliability for assessing pain intensity. Active modalities such as muscle strength exercises were found to be effective in reducing pain and disability, as well as improving quality of life. ${ }^{[11]}$

Early stage of presentation of knee OA contains pain with weight bearing activities such as climbing stairs and squatting, in later stages both pain and stiffness are reported after prolonged sitting.
${ }^{[12]}$ Symptoms of joint locking and bulking way may also occur with damage to stabilising menisci and ligaments which leads to increased risk of falls. ${ }^{[13]}$

There are two systemic reviews of studies designed to examine evidence of the effect of exercise in the management of knee OA describe support for aerobic exercise and strengthening exercises to reduce pain, improve balance and functional mobility. ${ }^{[14]}$ the Cochrane database of systematic reviews, ${ }^{[15]}$ the Philadelphia panel evidence-based clinical practise guidelines, ${ }^{[16]}$ and more recently a summary of systematic reviews of studies on physical therapy interventions for patients with knee $\mathrm{OA}$, ${ }^{[17]}$ indicated that there is evidence to support strengthening, stretching, and functional exercises as intervention for reducing the knee pain as a result of OA and to improve physical function. ${ }^{[17]}$

The Otago Exercise Program is an individually customized, home-based balance and strength fall prevention program. ${ }^{[5]}$ This programme is unique in its clearly defined prescription and ability to be readily implemented across the community. ${ }^{[18]}$ The program is designed to be performed for four weeks under the supervision of physical therapists. ${ }^{[19]}$ A recent systematic review of the literature concluded that multimodal exercises programs including muscle strengthening, gait, balance, coordination and functional exercises led to greater beneficial effects on balance and functional mobility than usual exercise program, at least in the short-term. ${ }^{[20]}$

A dual-task paradigm is a procedure in experimental neuropsychology that requires an individual to perform two tasks simultaneously, in order to compare performance with single-task conditions. ${ }^{[21]}$ The NSE group also given positive results by showing improvement in gait and balance function. These results are consistent with research suggesting that cognitive function is an indicator of gait and balance function. ${ }^{[22]}$ Dual task net step exercise is the task that require the simultaneous use of cognitive and gait 
functions this improves the mobility among the patients gait and body sway balance who underwent non motor cognitive dual tasking. The dual task principle uses a net and features slow and low-impact physical movement. ${ }^{[23]}$

For outcome measures are as follows: 1) Time Up and Go test and 2) Berg Balance Scale. ${ }^{[24]}$ Time Up and Go test is used to assess a person's mobility and requires Balance Scale is to measure balance among older people with impairment in balance function by assessing the performance of functional tasks which has a 14-item scale designed to measure the balance of the older adult. The TUG test was used to assess the participants' gait and -week balance follow-up. it is used frequently in the elderly population, as it is easy to administer and can generally be completed by older adults. Improvements in gait function were also evaluated using Timed Up and Go test scores.

The aim was to evaluate the effectiveness of Otago exercise Verses Dual task net step exercise on balance and functional mobility in community dwelling elderly person with osteoarthritis of knee and to Compare the effectiveness of Otago exercise Vs Dual task net step exercise on balance and functional mobility in community dwelling elderly person with osteoarthritis of knee by using BBS and TUG test.

\section{MATERIALS AND METHODS}

An approval for the study was obtained from the institutional Ethical Committee of College of Physiotherapy, Wanless Hospital, Miraj Medical Centre, Miraj. A Randomized controlled trial was conducted in subjects who are community dwelling elderly persons with knee OA in Miraj Medical Centre, Wanless Hospital, Miraj and old age home respectively. Sample was achieved by Simple random sampling. A Total of $n=36$ subjects were selected. All the subject were screened for inclusion criteria i.e. community dwelling elderly persons, Age group of 60-70 years, with knee OA. The exclusion criteria included of subjects with any neurological conditions, cardiorespiratory conditions, having/had joint replacement surgery or arthroscopic surgery on the affected side of the knee, other surgery on lower limbs within the past 6 months, severe deformity of lower limbs (e.g., knee varus or knee valgus). The Demographic data including age, gender, patient diagnosed with osteoarthritis of knee > six months, pain, knee OA (grading 2-3) Kellgren and Lawrence grading system, balance, functional mobility and other symptoms were collected through data sheet. All the subjects were given written consent prior to the intervention and were briefed about the study. Subjects were randomly divided into two groups with Group A of 18 and Group B of 18 subjects. Group A performed Otago exercises and Group B performed Dual task net step exercises for 4 weeks and 3 days per week. The data was collected pre and post treatment using visual analogue scale, berg balance scale, time up and go test.

\section{Procedure:}

\section{OTAGO EXERCISE: GROUP A- Protocol $=40 \mathrm{~min} /$ thrice in a week for 4 weeks.}

Lower extremity strengthening (each exercise is to be done slowly e.g. 2-3 seconds to lift the leg and 4-6 seconds to lower the leg and through the full functional range of motion. The goal is to perform each exercise for two sets of 10 repetitions. With person sitting in a hard, straight-back chair: performing 5 minutes of active, gentle warm-up exercises to minimize soreness. Have patient perform unilateral knee extension. Repeat for the opposite leg. With person in standing use a counter or heavy furniture to support as needed, have patient perform unilateral knee flexion. Repeat for the opposite leg. Have patient perform unilateral hip abduction. Repeat for the opposite leg. Raise up on toes to strengthen ankle plantarflexors. Rock back on heels for ankle dorsiflexors. Next is the Balance Training i.e. knee bends-10 repetitions, 
backward walking-10steps, 4 times, walking and turning around- Make figure of " 8 ", 2 times, sideways walking-10 steps, 4 times, tandem stance-10 seconds, tandem walk-10 steps, 2 times, heel walking-10 steps, 4 times, toe walking-10 steps, 4 times, sit to stand- 5 stands with two hands, with one hand, with no support

\section{DUAL TASK NET STEP EXERCISE: GROUP B- Protocol $=40 \mathrm{~min} /$ thrice in a week for 4 weeks.}

Fumanet is a $4 \mathrm{~m} \times 1.5 \mathrm{~m}$ net that comprises $50 \mathrm{~cm} \times 50 \mathrm{~cm}$ squares arranged in a $3 \times 8$ grid. The columns are labelled $\mathrm{A}$, $\mathrm{B}, \mathrm{C}$ and the rows are numbered 1 to 8 such that each of the 24 squares is assigned a unique coordinate. Step designs are provided by the instructors, who demonstrate the step designs to the participants. All participants are required to repeat the step designs in exactly the same way as their instructors.

\section{NSE program consisted of 3 stages.}

1. The first was the warm-up step, conducting at the beginning of each session to assess the participants' physical condition.

2. The second was the trial step, where participants learned the new step design to be performed during that session. The participants have to memorize each step design on the basis of the instructor's demo participants practiced each step design twice. The instructors tried to encourage the participants, never mentioning their mistakes.

3. The third stage was the recreation step. The participants formed a line and step in the same rhythm while singing a child and move in synchrony with the other participants while singing the song.

The total time for actual walking by each participant is approximately 20 minutes in each session. Throughout the 4week NSE program, the difficult the number of steps required in each session are gradually increased. Participants are expected to walk 216 of the program and 240 steps per session from the 3 to 4 weeks.
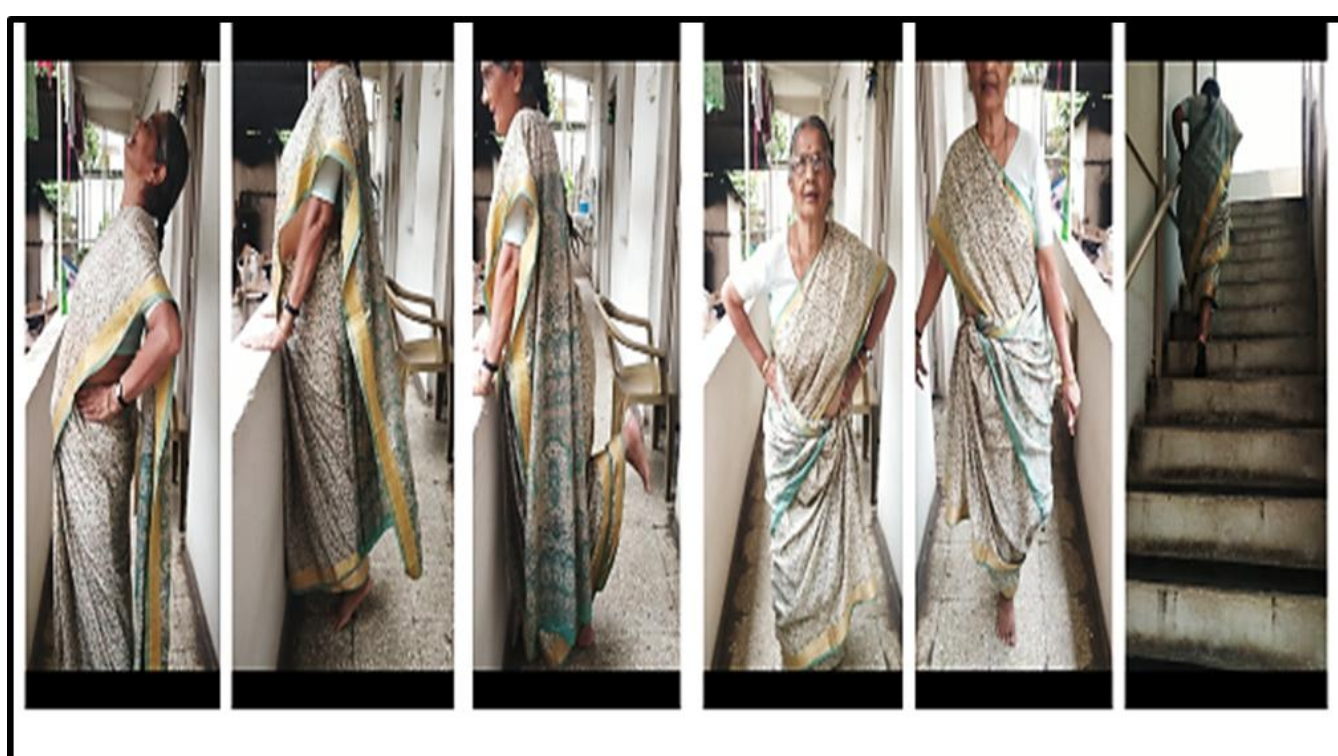

Fig. 1: Representation of the Otago exercises. 

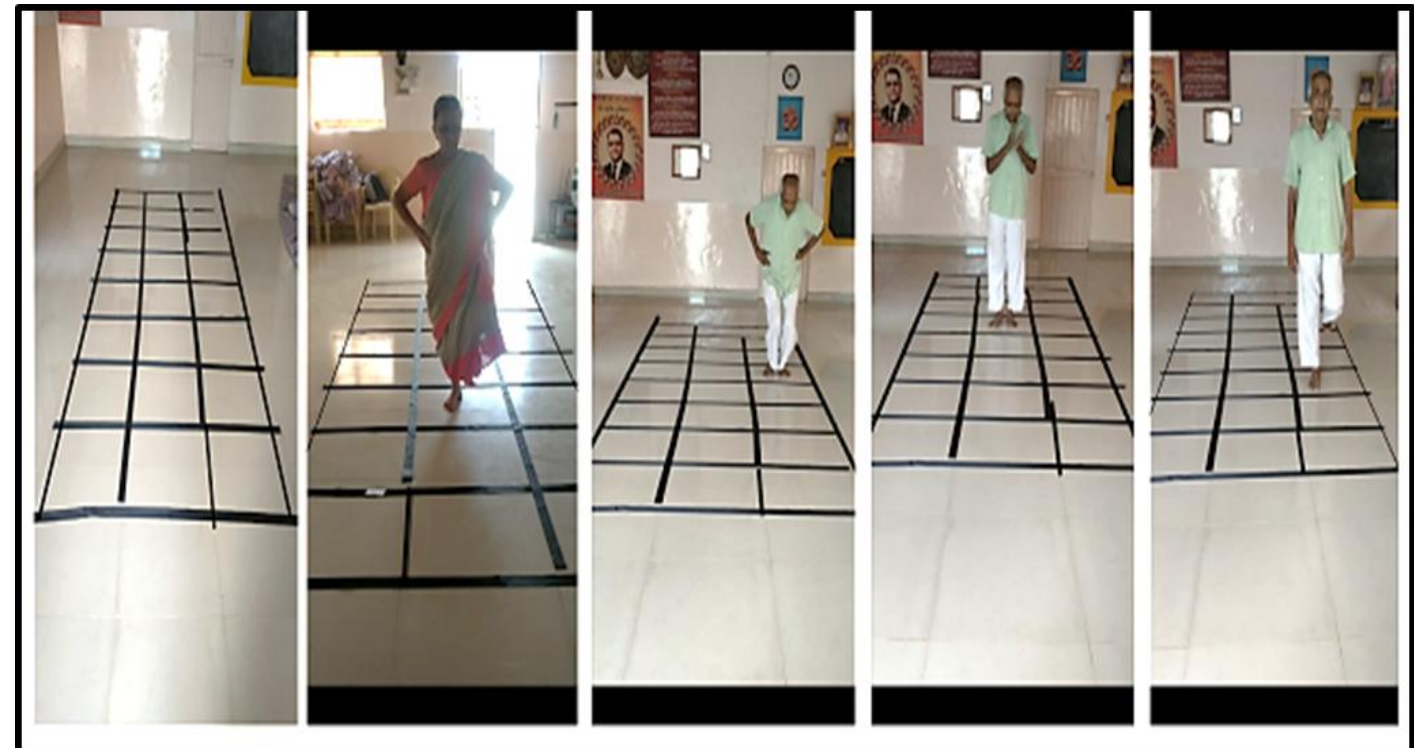

Fig. 2: Representation of Dual Task Net Step Exercises.

The assessment of the pain, balance and functional mobility was done by following scales:

PAIN: VAS (Visual Analogue Scale) Validity $=0.941 /$ Reliability $=0.97(0.96-$ 0.98): The pain intensity will be measured using Visual Analogue Scale. A $10 \mathrm{~cm}$ line will be drawn on which zero represents No pain and 10 represents highest pain intensity.

BALANCE: BBS (Berg-Balance Scale): It consists of 14 tasks challenging static and dynamic balance and is widely used in the clinical setting for a variety of patient populations. Effect of the Berg Balance Scale has been well documented for people who can ambulate independently in the community for people with knee OA, performance ranged from 50 to 56 out of 56 . One study showed a significant betweengroup difference (participants with knee OA scored 50/56 compared with 53/56 for healthy controls). [Inter-rater..98 and intrarater: 99 =reliability].

FUNCTIONAL MOBILITY: (Time Up And Go Test): The Timed Up and Go (TUG) test is functional mobility and requires both static and dynamic balance. [Intra-rater and inter-rater reliability $=0.97$ ]. Equipments used: Fumanet, supported chair, stopwatch, cone stopper, stationary.
STATISTICAL ANALYSIS: All statistical analysis was done by using SPSS 16.0 for windows. The level of significance was set at $p=0.05$. Descriptive analysis was used to calculate mean and standard deviation. Paired t test was performed for intragroup scores and independent $t$ test was performed for intergroups scores respectively.

\section{RESULTS}

The present study was done to find out the effect of otago exercises and dual task net step exercises on balance and functional mobility in community dwelling elderly person with knee OA. Total of 36 participants were evaluated. Demographic charateristics showed high number of females than males, age group was from 60 to 70 and mean age was $66.1 \pm 4.2$.

Table No.1: Mean age comparison in the two intervention groups:

\begin{tabular}{|l|l|l|l|l|}
\hline Groups & Mean & SD & Max & Min \\
\hline Group A & 66.16 & 4.29793 & 74 & 60 \\
\hline Group B & 67 & 4.24264 & 76 & 61 \\
\hline
\end{tabular}

Table No.2: Gender distribution in two intervention groups A and $B$

\begin{tabular}{|l|l|l|l|l|l|l|}
\hline Gender & $\begin{array}{l}\text { Group } \\
\text { A }\end{array}$ & \% & $\begin{array}{l}\text { Group } \\
\text { B }\end{array}$ & \% & Total & \% \\
\hline Male & 06 & 33.33 & 09 & 50 & 15 & 41.67 \\
\hline Female & 12 & 66.66 & 09 & 50 & 21 & 58.33 \\
\hline Total & 18 & 100 & 18 & 100 & 36 & 100 \\
\hline
\end{tabular}


Raymol Dagani Mohan Rao et.al. Effect of otago exercise versus dual task net step exercise on balance and functional mobility in community dwelling elderly person with knee osteoarthritis - a randomised control trial.

Table No. 3:_Descriptive statistics of group A receiving Otago exercises on community dwelling patients having knee osteoarthritis on balance and functional mobility via visual analogue scale(VAS), berg balance scale(BBS),time up and go test( TUG) using paired t test.

\begin{tabular}{|l|l|l|l|l|l|l|}
\hline Group & scale & $\mathbf{N}$ & Mean & Std. Deviation & Paired t statistic & P value \\
\hline \multirow{4}{*}{ Group A } & Pre VAS & 18 & 5.89 & .900 & 11 & .002 \\
\cline { 2 - 5 } & Post VAS & 18 & 4.06 & 1.162 & & \\
\cline { 2 - 5 } & Pre BBS & 18 & 34.39 & 6.818 & -6.008 & .000 \\
\cline { 2 - 5 } & Post BBS & 18 & 40.72 & 2.697 & & \\
\cline { 2 - 5 } & Pre TUG & 18 & 14.189 & .8930 & \multirow{2}{*}{14.067} & .008 \\
\cline { 2 - 5 } & Post TUG & 18 & 11.89 & .758 & & \\
\hline Group B & Pre VAS & 18 & 5.72 & .669 & 6.969 & .002 \\
\cline { 2 - 5 } & Post VAS & 18 & 4.61 & .698 & & .009 \\
\cline { 2 - 5 } & Pre BBS & 18 & 34.22 & 6.404 & -6.735 & \\
\cline { 2 - 5 } & Post BBS & 18 & 38.28 & 4.376 & & .094 \\
\cline { 2 - 5 } & Pre TUG & 18 & 14.478 & .8214 & & \\
\cline { 2 - 4 } & Post TUG & 18 & 13.11 & .900 & & \\
\hline
\end{tabular}

Table No. 4: Comparison between OTAGO EXERCISE and DUAL TASK NET STEP EXERCISE.

\begin{tabular}{|c|c|c|c|c|c|c|}
\hline \multicolumn{7}{|c|}{ Group comparison table } \\
\hline \multicolumn{2}{|l|}{ Variables } & Mean & Std. Deviation & $\mathbf{N}$ & CV & p value \\
\hline \multirow{2}{*}{ VAS Post } & Group A & 4.06 & 1.162 & 18 & 28.646 & \multirow[b]{2}{*}{.091 } \\
\hline & Group B & 4.61 & .698 & 18 & 15.13306 & \\
\hline \multirow[t]{2}{*}{ TUG Post } & Group A & 11.89 & .758 & 18 & 6.379026 & \multirow[b]{2}{*}{.000} \\
\hline & Group B & 13.11 & .900 & 18 & 6.866345 & \\
\hline \multirow[t]{2}{*}{ BBS Post } & Group A & 40.72 & 2.697 & 18 & 6.621751 & \multirow[b]{2}{*}{.052} \\
\hline & Group B & 38.28 & 4.376 & 18 & 11.43348 & \\
\hline
\end{tabular}

Thus alternative hypothesis $\left(\mathrm{H}_{1}\right)$, there is a significant difference in effect of Otago exercise and dual task net step exercises to improve balance and functional mobility in elderly patient with Osteoarthritis of knee. The study showed statistically significant difference in TUG and BBS in the functional mobility and balance. The coefficient variables are less in group A as compared to group B.

\section{DISCUSSION}

The present study was conducted on community dwelling elderly patients with knee OA in balance and functional mobility dysfunction in Otago exercise program and dual task net step exercise, which was evaluated on three different outcome measures. This includes 36 total number of patients. This study shows an idea that more number of female prone to balance issues and functional dysfunction compare to males. Because of the decreased muscles quadriceps strength which has been associated with increased pain, cartilage loss and reduced physical functions along with increase in the weight of the body. Although the rate of torque development and acceleration time provide information about fast muscle contraction capacity, the nature of the muscle action differs (dynamic versus isometric), which may explain the discrepancy of the results.

In addition, only women participated in the present study, and women are more prone to present a greater decline in muscle function than men. In my study for the assessment of balance and functional mobility we are using 1) Time Up and Go test 2) Berg Balance Scale. TUG test It is used to assess a person's mobility and requires both static and dynamic balance. Berg Balance Scale is to measure balance among elderly people with balance dysfunctions by assessing the performance of functional tasks which has 14-items scale designed to measure the balance of the older adults.

The first objective of the study was to investigate the effect of Otago exercises on balance and functional mobility on community dwelling elderly person having knee OA. Results indicated a significant difference between the two groups regarding pain, balance and functional mobility. Shraddha et al conducted an experimental study on both male and female on balance and risk of fall in the community dwelling individuals having knee OA. The exercise group did 4 weeks of Otago exercises. Then according to the findings this program is effective in increasing 
strength and balance. Thus is effective in reducing the risk of fall in the older adult. Spink et al reported that exercises that focused on muscles of ankles and feet were considered to be important components of successful podiatry fall prevention trial.

Otago showed increased strength in the lower limbs and also improved balance. They were improved by walking and turning around, heel to toe walking and stair walking. As we know that OA is the most common joint disease that affects the elderly and ageing is one of the most important etiology for the disease. Strengthening and balance exercises mainly of lower limb strengthening are effectual in the prevention of fall. Both the static and dynamic balance is needed. Due to which it is ability to maintain the projection of body's center of mass within manageable limits of base of support is essential. Diminished strength is a well-documented finding in the older adult and the effects of aging are considered, systems like sensory, central processing, neural pathways for motor control and musculoskeletal system enabled the contribution of physiological systems to be evaluated.

The second objective of the study was to investigate the effect of Dual Task Net Step exercise on balance and functional mobility on community dwelling elderly person having knee OA. The dual task net step exercise was done on the community dwelling elderly persons with knee OA for the first time as this condition causes dysfunction in balance and functional mobility. Previous studies i.e $\mathrm{Li}$ et al observed improvement in both cognitive and motor function such as gait and bodysway balance in older adults who underwent nonmotor cognitive dual task training. And the other studies had also proven that cognitive function is an indicator of gait and balance function i.e. the dual task net step exercise showed improvement in gait and balance function. Multifaceted neuropsychological influences on walking and interactions with mobility control are increasingly reported in studies and emphasize the link between cognitive and motor functions. Dual task net step exercise is a new exercise intervention in balance training and not may have been conducted. The correlation between cognitive function on improving balance by dual task net step exercises which mainly focuses on the cognitive health and also this function is an indicator of gait and balance function. As previous studies have stated that gait is no longer considered to be merely an automated motor activity and it is understood that to involve a higher level of cognitive function.

After the completion of my study we observed that both the exercises showed significant improvement. The main objective of the study was to compare the effect of Otago exercise verses Dual task net step exercise in community dwelling elderly persons with knee OA. So the study results showed a significant difference between the two group where the Otago exercise was more effective and useful in the treatment for community dwelling elderly persons with knee OA having issues on balance and functional mobility.

\section{CONCLUSION \& LIMITATIONS}

According to the findings of the study we concluded that both the groups showed improvement in balance function and functional mobility. The study showed statistically significant difference in TUG and BBS in the functional mobility and balance. The coefficient variables are less in group A as compared to group B. Hence, the study concluded that Otago exercise are more effective than the Dual Task Net Step Exercise in improving balance and functional mobility in community dwelling elderly persons with knee OA respectively. Small sample size was included in the study.

The improvements acquired by the community dwelling elderly persons were not traced for longer duration, therefore cannot mention about the enduring effect of both the exercises. 


\section{ACKNOWLEDGEMENT}

It's my great pleasure and privilege to express my deep- felt gratitude to our respected Principal sir Dr. Ronald Prabhakar and Guide Dr. Archana D. Methe who immensely helped me and rendered their advice, precious time, constant encouragement, knowledge and relevant information regarding my study, and whose suggestion and guidance has enlightened me on this subject. I am also grateful to Dr. Amit Nagrale, Professor, Dept. Of community medicine, Miraj Medical Centre, College of Physiotherapy, Wanless Hospital, for help in the data analysis of this study. I give my sincere gratitude to my subjects those who voluntarily participated in my study.

Above all, I would like to thank my parents for their blessings, love, constant support, affection and encouragement.

Praise and glory to the God almighty who is the source of strength, foundation of my knowledge and the source of inspiration in every walk of life.

\section{Conflict of Interest: None}

\section{Source of Funding: None}

\section{Ethical Approval: Approved}

\section{REFERENCES}

1. Egloff $\mathrm{C}$, Hügle $\mathrm{T}$, Valderrabano $\mathrm{V}$. Biomechanics and pathomechanisms of osteoarthtritis, Swiss Med Wkly 2012.

2. Pal C, Singh P, Chaturvedi S, Pruthi K, Vij A. Epidemiology of knee osteoarthritis in India and related factors. Indian Journal of Orthopaedics. 2016; 50(5):518.

3. ShyamGanvir, Zambare BR. A study to determine the prevalence of osteoarthritis of knee in rural Maharashtra. International journal of recent trends in science and technology. 2015;16(2):428-31.

4. Jadhav VS, Anap DB. Effectiveness of Mulligan Mobilization Versus Mckenzie Exercises in Knee Osteoarthritis: A Single Blind Randomized Controlled Trial. Physiotherapy Rehabilitation 2019;4(177):2.

5. Dr. Deepti Wadhwa and Dr. Deepali Hande: Effectiveness of the Otago Exercise
Program on Reducing the Fall Risk in Elderly: Single Case Report. Imperial Journal of Interdisciplinary Research(IJIR)Vol-2,Issue6,2016,ISSN:24541362,http://www.onlinejo urnal.in

6. Hinman RS, Bennell KL, Metcalf BR, Crossley KM. Balance impairments in individuals with symptomatic knee osteoarthritis: a comparison with matched controls using clinical tests. Rheumatology 2002

7. Tinetti ME, Speechley M. The effect of falls and fall injuries on functioning in community-dwelling older persons. $J$ Gerontol 1998

8. Berenbaum, F: Osteoarthritis, epidemiology, pathology, and pathogenesis. In Klippel, JH, et al (eds): prime on the rheumatic diseases, ed. 12. Atlanta: Arthritis Foundation,2001.

9. Roddy, E, et al: Evidence-based recommendations for the role of exercise in the management of osteoarthritis of the knee- the MOVE consensus.

10. Roddy, E, Zhang, W, and Doherty, M: Aerobic walking or strengthening exercise for osteoarthritis of the knee? A systematic review.

11. Fransen, M, McConnell, S, and Bell, M: Exercise for osteoarthritis of the hip and knee. The Cochrane Database of Systematic Reviews.2001.

12. Philadelphia Panel Evidence-based Clinical Practice Guidelines on Selected Rehabilitation Interventions for knee Pain. Physiotherapy.

13. Jamtvedt, G, et al: Physical therapy interventions for patients with osteoarthritis of the knee; an overview of systematic reviews. Physical Therapy,2008.

14. Gardner, MM, et al: Practical implementation of an exercise-based falls prevention programme. Age Ageing,2001.

15. Howe, TE, et al; Exercise for improving balance in older people. Cochrane Database Syst Rev ,2007.

16. Kevin M Means and Patrick M. Kortebein: Geriatrics: Musculoskeletal: Osteoarthritispage no 120.

17. Mulligan RK, Hinman RS, Kasza J, Bennell KL. Effectiveness of internet-delivered education and home exercise supported by behaviour change SMS on pain and function for people with knee osteoarthritis: a randomised controlled trial protocol. BMC 
Raymol Dagani Mohan Rao et.al. Effect of otago exercise versus dual task net step exercise on balance and functional mobility in community dwelling elderly person with knee osteoarthritis - a randomised control trial.

musculoskeletal disorders. 2019 Dec;20(1):342.

18. Felson, DT: Developments in the clinical understanding of osteoarthritis. Arthritis Res her 11:203, 2009. Retrieved May 15,2011, fromhttp://arthritisresearch.com/content/11/ $1 / 203$.

19. Dr. Deepti Wadhwa and Dr. Deepali Hande: Effectiveness of the Otago Exercise Program on Reducing the Fall Risk in Elderly: Single Case Report. Imperial Journal of Interdisciplinary Research(IJIR) Vol-2,Issue-6,2016,ISSN:2454-1362, http://www.onlinejournal.in

20. Li KZ ,Roudaia E , Lussier M , Bherer L, Leroux A , McKinley PA . Benefits of cognitive dual-task training on balance performance in healthy older adults. $\mathbf{J}$ Gerontol A Biol Sci Med Sci. 2010; 65 (12): 1344-1352.

21. A dual-task paradigm, assessed on $12 / 02 / 2017$ at 6:00 pm https://en.wikipedia.org/wiki/Dualtask_paradigm. page modified on 17 December 2016, at 07:31
22. Bhodaji S. Dual-Task Net-Step Exercise Versus Balance Training Exercise Program On Foam Rubber Pad In Community-Based Older Adults: A Randomized Clinical Trial (Doctoral dissertation, KLE University, Belagavi, Karnataka).2017

23. Salekar S, Khandale SR. Effect of Otago exercise program on balance and risk of fall in community-dwelling individuals having knee osteoarthritis. S Salekar, SR Khandale - 2019 - ijarnd.com.

24. Kitazawa K, Showa S, Hiraoka A, Fushiki Y, Sakauchi H, Mori M. Effect of a dual task net-step exercise on cognitive and gait function in older adults. Journal of geriatric physical therapy. $2015 \mathrm{Jul}$ 1;38(3):133-40.

How to cite this article: Rao RDM, Methe AD, Patil $\mathrm{H}$ et.al. Effect of Otago exercise versus dual task net step exercise on balance and functional mobility in community dwelling elderly person with knee osteoarthritis - a randomised control trial. Int J Health Sci Res. 2021; 11(7):179-187. DOI: https://doi.org/10. 52403/ijhsr.20210726 